\title{
A Novel Ensemble Learning Approach for Corporate Financial Distress Forecasting in Fashion and Textiles Supply Chains
}

\author{
Gang Xie, ${ }^{1}$ Yingxue Zhao, ${ }^{2}$ Mao Jiang, ${ }^{1}$ and Ning Zhang ${ }^{1}$ \\ ${ }^{1}$ Academy of Mathematics and Systems Science, Chinese Academy of Sciences, Beijing 100190, China \\ ${ }^{2}$ School of International Trade and Economics, University of International Business and Economics, Beijing 100029, China \\ Correspondence should be addressed to Gang Xie; gxie@amss.ac.cn
}

Received 23 December 2012; Revised 19 January 2013; Accepted 25 January 2013

Academic Editor: Tsan-Ming Choi

Copyright (c) 2013 Gang Xie et al. This is an open access article distributed under the Creative Commons Attribution License, which permits unrestricted use, distribution, and reproduction in any medium, provided the original work is properly cited.

\begin{abstract}
This paper proposes a novel ensemble learning approach based on logistic regression (LR) and artificial intelligence tool, that is, support vector machine (SVM) and back-propagation neural networks (BPNN), for corporate financial distress forecasting in fashion and textiles supply chains. Firstly, related concepts of LR, SVM, and BPNN are introduced. Then, the forecasting results by LR are introduced into the SVM and BPNN techniques which can recognize the forecasting errors in fitness by LR. Moreover, empirical analysis of Chinese listed companies in fashion and textile sector is implemented for the comparison of the methods, and some related issues are discussed. The results suggest that the proposed novel ensemble learning approach can achieve higher forecasting performance than those of individual models.
\end{abstract}

\section{Introduction}

In fashion and textile industry, there is a great deal of change due to global sourcing and high levels of price competition. In addition, fashion and textiles have market characteristics such as short product lifecycle, high volatility, low predictability, and a high level of impulse purchase [1-3]. The rigorous competition and rapid change demand cause financial risk to corporations in a fashion and textile supply chain [4-8]. As financial risk may be infectious from one corporation to another within the supply chain, the prediction of corporate financial distress is important to fashion and textile supply chain management.

For better performance in fashion retailing, more and more research has been focused on forecasting, including sales forecasting [9-11], fashion retail forecasting [12], and color trend forecasting $[13,14]$. However, in fashion and textile sector, little attention has been paid to corporate financial distress forecasting, which is important to various stakeholders (i.e., management, investors, employees, shareholders, and other interested parties) as it provides them with timely warnings. From a managerial perspective, financial distress forecasting tools allow for taking timely strategic actions so that financial distress can be avoided [15].

Many traditional techniques have been presented to predict corporate financial distress, including univariate approaches [16], linear multiple discriminant approaches (MDA) $[17,18]$, multiple regression $[19,20]$, logistic regression [21], and factor analysis [22]. However, strict assumptions of traditional statistics such as linearity, normality, and independence among predictor variables limit their applications in the real world [23].

Due to limitations of traditional statistical and econometric models, some nonlinear and artificial intelligence (AI) models, including neural networks [24], case-based reasoning $[25,26]$, and support vector machine [27], have been used for corporate financial distress forecasting. However, individual forecasting methods have limited capability in the description of financial characteristics. In particular, to some complex forecasting problems, there may be a bias in the results when only an individual method is used [28]. A more appropriate approach for improving the forecasting accuracy is the combination of individual methods, which always performs better than the worst individual model 
TABLE 1: Definition of variables.

\begin{tabular}{ll}
\hline & $\begin{array}{l}\text { Rate of return on common stockholders' } \\
\text { equity }\left(x_{1}\right)\end{array}$ \\
& Rate of return on total assets $\left(x_{2}\right)$ \\
& Earn per share $\left(x_{3}\right)$ \\
& Main operating margins $\left(x_{4}\right)$ \\
\hline \multirow{3}{*}{ Operating ability } & Turnover rate of total assets $\left(x_{5}\right)$ \\
& Turnover rate of account receivable $\left(x_{6}\right)$ \\
& Turnover rate of inventory $\left(x_{7}\right)$ \\
\hline Debt-repaying ability & $\begin{array}{l}\text { Cash ratio }\left(x_{8}\right) \\
\text { Acid test ratio }\left(x_{10}\right)\end{array}$ \\
& Current ratio $\left(x_{11}\right)$ \\
\hline Growing ability & Total assets growth rate $\left(x_{12}\right)$ \\
\hline
\end{tabular}

on predictions and sometimes can outperform the best individual model [29].

Some hybrid methods have been used for corporate financial distress forecasting [30]. In terms of experimental results, AdaBoost ensemble with single attribute test (SAT) outperforms AdaBoost ensemble with decision tree (DT), single DT classifier, and single support vector machine (SVM) classifier. As a conclusion, the choice of weak learner is crucial to the performance of AdaBoost ensemble, and AdaBoost ensemble with SAT is more suitable for corporate financial distress forecasting of Chinese listed companies [31]. Also, empirical results indicate that the integration of principal component analysis (PCA) with MDA can produce better performance in short-term financial distress forecasting of Chinese listed companies [32]. However, there is not an ensemble learning approach that can improve the forecasting performance in one method by recognizing Type I error and Type II error in another method yet.

This paper proposes a novel ensemble learning approach based on logistic regression (LR) and artificial intelligence tool, that is, support vector machine (SVM) and backpropagation neural networks (BPNN) for the prediction of corporate financial distress in fashion and textiles supply chains. Firstly, related concepts of LR, SVM, and BPNN are introduced. Then, the forecasting results by LR are introduced into the SVM/BPNN technique as an ensemble approach. Empirical analysis is implemented for the comparison of the methods, and some related issues are discussed.

The rest of this paper is organized as follows. The basic concepts of LR, SVM, and BPNN are introduced in Section 2. We describe our proposed ensemble learning approach in Section 3. Section 4 presents empirical analysis to illustrate the proposed approaches and some related issues, including performance comparison and analysis. Finally, we make a conclusion and discuss future research in Section 5.

\section{Related Concepts}

In this section, concepts of LR, SVM, and BPNN are introduced as follows.
2.1. Logistic Regression Model. In a logistic regression model (LR), dependent variable is always in categorical form and has two or more levels [33]. In this study, we consider the situation where we observe a binary outcome variable $y$ and a vector $x=\left(1, x_{1}, x_{2}, \ldots, x_{k}\right)$ of covariates for each of $n$ individuals. We code the two class via a $0 / 1$ response $y_{i}$, where $y_{i}=1$ is for the first class (financial distress) and $y_{i}=0$ is for the second one (no financial distress). Let $p$ be the conditional probability associated with the first class. In a logistic regression model, probability $p$ of the dichotomous outcome event is related to a set of explanatory variables $x$ as follows:

$$
\operatorname{Logit}(p)=\ln \left(\frac{p}{1-p}\right)=f(x, \beta)=\beta^{T} x,
$$

where $\beta=\left(\beta_{0}, \beta_{1}, \beta_{2}, \ldots, \beta_{k}\right)$ is the coefficient vector of the model and $\beta^{T}$ is the transpose vector. Equation (1) is logit transformation and $p /(1-p)$ is odds ratio.

Let $D=\left\{\left(x_{i}, y_{i}\right): i=1,2, \ldots, n\right\}$ be the training data set, which is a set of independent and identically distributed random variables. The regression coefficient $\beta_{i}$ estimated from the data is interpretable as log-odds ratios or, in term of $\exp \left(\beta_{i}\right)$, as odds ratios. The log-likelihood for $n$ observations is used for estimating regression coefficients $\beta_{i}$ as follows:

$$
\beta_{i}=\sum_{i=0}^{n}\left\{y_{i} \beta^{T} x_{i}-\log \left(1+e^{\beta^{T} x_{i}}\right)\right\}
$$

where $e^{\beta^{T}}$ gives odds ratio and this value reflects the effect of indicators in financial distress.

2.2. Support Vector Machine. Support vector machine (SVM), proposed by Vapnik, has been proved to possess excellent capability for classification [34]. The conventional SVM achieves classification by mapping the input vectors on to a high-dimensional feature space and by then constructing a linear model that implements nonlinear class boundaries in the original space. The SVM employs an algorithm that finds a special kind of linear model, that is, the optimal hyperplane, which refers to the maximum-margin hyperplane and yields the maximum separation between decision classes. Thus, the optimal hyperplane separates the training examples with the maximum distance from the separating hyperplane to the closest training data samples. The training examples closest to the maximum-margin hyperplane are called support vectors. All other training examples, other than the support vectors, are useless for constructing the optimal hyperplane. As a result, it is possible for SVM models to effectively perform binary classification with a small size of training samples [28].

For the linearly separable case, a hyperplane, which separates the binary decision classes in the case of $n$ attributes, can be represented as the following equation:

$$
y=w_{0}+\sum_{i=1}^{n} w_{i} x_{i}
$$

where $y$ is the outcome, $x_{i}$ is the attribute value $(i=1, \ldots, n)$, and $w_{i}$ is the weight of $x_{i}$ learned by the algorithm. In (3), 
TABLE 2: Data of the sample set.

\begin{tabular}{|c|c|c|c|c|c|c|c|c|c|c|c|c|}
\hline$y$ & $x_{1}$ & $x_{2}$ & $x_{3}$ & $x_{4}$ & $x_{5}$ & $x_{6}$ & $x_{7}$ & $x_{8}$ & $x_{9}$ & $x_{10}$ & $x_{11}$ & $x_{12}$ \\
\hline 0 & 0.0763 & 0.0610 & 0.1900 & 0.1770 & 0.0033 & 0.0610 & 0.0613 & 0.1221 & 0.2987 & 0.0113 & 0.0094 & 0.0560 \\
\hline 0 & 0.0108 & 0.0198 & 0.1200 & 0.0290 & 0.0048 & 0.1280 & 0.0230 & 0.0960 & 0.5490 & 0.0096 & 0.0048 & 0.0365 \\
\hline 0 & 0.0151 & 0.0239 & 0.0400 & 0.1220 & 0.0015 & 0.0600 & 0.0118 & 0.8196 & 0.1210 & 0.0414 & 0.0300 & 0.0924 \\
\hline 1 & 0.0156 & -0.0501 & -0.4500 & -0.0030 & 0.0017 & 0.0343 & 0.0241 & 0.9500 & 0.5670 & 0.0134 & 0.0061 & -0.1970 \\
\hline 0 & 0.1275 & 0.0726 & 0.7300 & 0.1246 & 0.0043 & 0.1786 & 0.0193 & 0.2430 & 0.7925 & 0.0066 & 0.0031 & 0.6109 \\
\hline 0 & 0.0784 & 0.0538 & 0.3300 & 0.0627 & 0.0060 & 0.1306 & 0.0376 & 0.1330 & 0.5649 & 0.0065 & 0.0049 & 0.0606 \\
\hline 0 & 0.2256 & 0.0425 & 0.1400 & 0.0516 & 0.0045 & 0.1010 & 0.0293 & 0.1840 & 0.4229 & 0.0108 & 0.0064 & 0.4768 \\
\hline 1 & 0.0104 & 0.0038 & 0.1700 & 0.0054 & 0.0032 & 0.0197 & 0.0166 & 0.0360 & 0.3790 & 0.0133 & 0.0079 & -0.2854 \\
\hline 0 & 0.0725 & 0.0883 & 0.3200 & 0.1230 & 0.0052 & 0.0884 & 0.0210 & 0.1086 & 0.1470 & 0.0469 & 0.0360 & 0.3710 \\
\hline 0 & 0.0961 & 0.0755 & 0.7200 & 0.0410 & 0.0108 & 0.1027 & 0.0469 & 0.0912 & 0.4960 & 0.0144 & 0.0103 & 0.2070 \\
\hline 0 & 0.2951 & 0.3183 & 0.5600 & 0.2220 & 0.0091 & 0.0495 & 0.0513 & 0.0812 & 0.4670 & 0.0167 & 0.0128 & 0.8194 \\
\hline 1 & -0.2065 & -0.0239 & -0.6700 & -0.1801 & 0.0025 & 0.0297 & 0.0153 & 0.0214 & 0.7680 & 0.0057 & 0.0033 & 0.0289 \\
\hline 0 & 0.0267 & 0.0291 & 0.0700 & 0.0248 & 0.0052 & 0.1846 & 0.0269 & -0.1190 & 0.5268 & 0.0124 & 0.0076 & 0.0277 \\
\hline 0 & 0.0511 & 0.0446 & 0.1800 & 0.0347 & 0.0107 & 0.0905 & 0.0901 & 0.0702 & 0.2570 & 0.0270 & 0.0236 & 0.3270 \\
\hline 0 & 0.0456 & 0.0384 & 0.2500 & 0.0660 & 0.0033 & 0.0969 & 0.0076 & -0.0270 & 0.4795 & 0.0167 & 0.0078 & 0.1797 \\
\hline 1 & -0.6343 & -0.8480 & -0.7800 & -0.1544 & 0.0002 & 0.0048 & 0.0095 & -0.0858 & 0.6199 & 0.0017 & 0.0014 & -0.2280 \\
\hline 0 & 0.1319 & 0.1132 & 0.5000 & 0.0880 & 0.0061 & 0.0461 & 0.0257 & 0.0965 & 0.5983 & 0.0105 & 0.0070 & 0.3730 \\
\hline 0 & 0.1264 & 0.1365 & 0.2800 & 0.0701 & 0.0115 & 0.1525 & 0.0572 & 0.0680 & 0.6140 & 0.0080 & 0.0053 & 0.1741 \\
\hline 0 & 0.0860 & 0.0554 & 0.6400 & 0.1130 & 0.0050 & 0.0503 & 0.0128 & 0.1040 & 0.4122 & 0.0166 & 0.0105 & 0.6068 \\
\hline 1 & -0.1326 & -0.0199 & -0.5700 & -0.3669 & 0.0012 & 0.0085 & 0.0126 & 0.1151 & 0.9496 & 0.0059 & 0.0044 & -0.0895 \\
\hline 0 & 0.0429 & 0.0374 & 0.2000 & 0.0570 & 0.0030 & 0.0393 & 0.0275 & 0.1067 & 0.6500 & 0.0093 & 0.0071 & 0.1070 \\
\hline 0 & 0.0259 & 0.0380 & 0.1600 & 0.0267 & 0.0047 & 0.0757 & 0.0231 & 0.0600 & 0.5230 & 0.0097 & 0.0057 & 0.1204 \\
\hline 0 & 0.1319 & 0.0891 & 0.5000 & 0.0880 & 0.0061 & 0.0461 & 0.0257 & 0.0960 & 0.6000 & 0.0105 & 0.0070 & 0.8305 \\
\hline 1 & 0.2490 & -0.0314 & -0.4300 & -0.9043 & 0.0022 & 0.0200 & 0.0066 & 1.1200 & 0.8 & 0.0103 & 0.0062 & -0.0087 \\
\hline 0 & 0.1531 & 0.2057 & 0.5900 & 0.1046 & 0.0148 & 0.1115 & 0.0225 & 0.0745 & 0.3946 & 0.0223 & 0.0148 & 0.8600 \\
\hline 0 & 0.0458 & 0.0449 & 0.3100 & 0.0980 & 0.0024 & 0.0160 & 0.0139 & 0.1020 & 0.5500 & 0.0449 & 0.0458 & 0.2020 \\
\hline 0 & 0.0373 & 0.0414 & 1.6200 & 0.0834 & 0.0037 & 0.0233 & 0.0246 & 0.1980 & 0.1030 & 0.0865 & 0.0735 & -0.0475 \\
\hline 1 & -0.0697 & -0.0270 & -0.5700 & 0.1307 & 0.0024 & 0.0176 & 0.0081 & 0.0468 & 0.4403 & 0.0141 & 0.0084 & 0.2190 \\
\hline 0 & 0.2975 & 0.2130 & 0.6100 & 0.0803 & 0.0240 & 0.6377 & 0.0590 & 0.0711 & 0.6400 & 0.0092 & 0.0057 & 0.7032 \\
\hline 0 & 0.0462 & 0.0427 & 0.2700 & 0.0754 & 0.3400 & 0.0345 & 0.0223 & 0.0640 & 0.4870 & 0.0106 & 0.0080 & 0.1002 \\
\hline 0 & 0.0562 & 0.0605 & 0.4500 & 0.0369 & 0.0034 & 47 & 0.0242 & 0.0295 & .3440 & 0.0210 & 0.0177 & -0.0348 \\
\hline 1 & -0.3361 & -0.1000 & -0.4900 & 0.5420 & 0.0020 & 0.0170 & 0.0047 & 0.0845 & 0.5760 & 0.0123 & 0.0057 & 0.2414 \\
\hline 0 & 0.0368 & 0.0373 & 0.2200 & 0.0890 & 0.0040 & 0.0543 & 0.0265 & 0.1300 & 0.4790 & 0.0086 & 0.0055 & 0.0306 \\
\hline 0 & 0.0647 & 0.0759 & 0.2300 & 0.1170 & 0.0050 & 0.2683 & 0.0202 & 0.2110 & 0.1849 & 0.0396 & 0.0278 & 0.5052 \\
\hline 0 & -0.0844 & -0.0413 & 1.0700 & -0.0501 & 0.0109 & 0.1038 & 0.0618 & 0.0100 & 0.6498 & 0.0093 & 0.0070 & 0.1459 \\
\hline 1 & -0.0740 & -0.0329 & -0.5100 & 0.1145 & 0.0031 & 0.0222 & 0.0254 & -0.1030 & 0.3406 & 0.0160 & 0.0122 & 0.1555 \\
\hline 0 & 0.0353 & 0.0276 & 0.3600 & 0.0172 & 0.0116 & 0.1321 & 0.1134 & 0.0035 & 0.4608 & 0.0168 & 0.0140 & 0.0155 \\
\hline 0 & 0.0100 & 0.0129 & 0.0300 & 0.0040 & 0.0052 & 0.0469 & 0.0148 & 0.0640 & 0.6397 & 0.0100 & 0.0051 & -0.1158 \\
\hline 0 & 0.0697 & 0.0972 & 0.8800 & 0.1283 & 0.0046 & 0.0914 & 0.0168 & 0.0488 & 0.2510 & 0.0558 & 0.0465 & 0.6702 \\
\hline 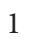 & -0.0164 & -0.9775 & 0.0300 & -0.0373 & 0.0030 & 0.0287 & 0.0299 & 0.0676 & 0.0928 & 0.0651 & 0.0810 & 0.0183 \\
\hline 0 & 0.0675 & 0.0711 & 0.3600 & 0.0890 & 0.0060 & 0.0643 & 0.0331 & 0.0690 & 0.0025 & 0.0353 & 0.0272 & 0.6003 \\
\hline 0 & 0.1286 & 0.0988 & 0.7400 & 0.1502 & 0.0049 & 0.1340 & 0.0361 & 0.0870 & 0.0314 & 0.0601 & 0.0233 & -0.7820 \\
\hline 0 & 0.0095 & 0.0089 & 0.2100 & 0.0047 & 0.0049 & 0.0769 & 1.3050 & 0.1180 & 0.7420 & 0.9100 & 0.4370 & -0.1620 \\
\hline 1 & -0.5781 & -0.0467 & -0.5700 & -0.1924 & 0.0038 & 0.0991 & 0.0340 & 0.0012 & 0.5180 & 0.0087 & 0.0072 & -0.0439 \\
\hline 0 & 0.0517 & 0.0591 & 0.6200 & 0.0007 & 0.0063 & 0.0371 & 0.0232 & -0.2009 & 0.2620 & 0.0255 & 0.0201 & -0.0660 \\
\hline 0 & 0.1323 & 0.0988 & 0.9000 & 0.1397 & 0.0049 & 0.0440 & 0.0228 & 0.1470 & 0.4730 & 0.0179 & 0.0135 & 0.1742 \\
\hline 0 & 0.0067 & 0.0117 & 0.0200 & 0.0250 & 0.0013 & 0.0509 & 0.0138 & 0.0910 & 0.5044 & 0.0126 & 0.0113 & 0.0357 \\
\hline 1 & -0.1459 & -0.0001 & -0.7800 & -0.0456 & 0.0046 & 0.0850 & 0.0255 & -0.1059 & 0.6859 & 0.0085 & 0.0058 & 0.1287 \\
\hline 0 & 0.0199 & 0.0223 & 0.1200 & 0.0294 & 0.0046 & 0.0774 & 0.0526 & 0.0703 & 0.2575 & 0.0160 & 0.0126 & 0.0919 \\
\hline 0 & 0.0756 & 0.0626 & 0.4800 & 0.0787 & 0.0052 & 0.0468 & 0.0117 & 0.0200 & 0.4694 & 0.0161 & 0.0064 & 0.1685 \\
\hline
\end{tabular}


TABLE 2: Continued.

\begin{tabular}{ccccccccccccc}
\hline$y$ & $x_{1}$ & $x_{2}$ & $x_{3}$ & $x_{4}$ & $x_{5}$ & $x_{6}$ & $x_{7}$ & $x_{8}$ & $x_{9}$ & $x_{10}$ & $x_{11}$ & $x_{12}$ \\
\hline 0 & 0.0319 & 0.0448 & 0.1600 & 0.0520 & 0.0053 & 0.0586 & 0.0413 & 0.1119 & 0.5785 & 0.0631 & 0.0504 & 0.1154 \\
1 & 0.1256 & 0.0158 & 0.1500 & -0.0792 & 0.0018 & 0.0399 & 0.0159 & 0.1130 & 0.0620 & 0.0078 & 0.0005 & 0.0186 \\
0 & -0.2245 & 0.0051 & -0.1400 & -0.0513 & 0.0045 & 0.0491 & 0.0340 & 0.0781 & 0.8475 & 0.0045 & 0.0029 & -0.0525 \\
0 & 0.0605 & 0.0577 & 0.2700 & 0.0636 & 0.0067 & 0.0987 & 0.0448 & 0.0852 & 0.2997 & 0.0122 & 0.0076 & 0.2740 \\
0 & 0.0939 & 0.1019 & 0.9500 & 0.2602 & 0.0037 & 0.1154 & 0.0098 & 0.1597 & 0.1496 & 0.0572 & 0.0473 & 0.1090 \\
1 & -0.1652 & -0.1967 & -2.1300 & -0.0291 & 0.0007 & 0.0193 & 0.0009 & 0.4450 & 0.9094 & 0.0117 & 0.0094 & -0.4476 \\
0 & 0.0361 & 0.0426 & 0.5500 & 0.0339 & 0.0068 & 0.0778 & 0.0217 & 0.0335 & 0.4201 & 0.0164 & 0.0095 & 0.1240 \\
0 & 0.0086 & 0.0163 & 0.0300 & 0.0102 & 0.0052 & 0.0747 & 0.0308 & 0.0350 & 0.4217 & 0.0123 & 0.0080 & 0.0984 \\
0 & 0.0135 & 0.0208 & 0.0400 & 0.0147 & 0.0033 & 0.0902 & 0.0361 & 0.1088 & 0.4901 & 0.0083 & 0.0067 & 0.0711 \\
1 & 0.1437 & 0.1113 & 0.3700 & 0.1548 & 0.0061 & 0.0269 & 0.0200 & 0.0856 & 0.4080 & 0.0197 & 0.0197 & 1.5970 \\
\hline
\end{tabular}

TABLE 3: Training set and testing set.

\begin{tabular}{lccc}
\hline Sample & Distressed set & Non-distressed set & Total \\
\hline Training set & 10 & 30 & 40 \\
Testing set & 5 & 15 & 20 \\
\hline Total & 15 & 45 & 60 \\
\hline
\end{tabular}

the weights are the parameters that determine the hyperplane. By using the support vectors, SVM models approximate the maximum-margin hyperplane as follows:

$$
y=b+\sum_{i=1}^{n} \alpha_{i} y_{i} \mathbf{x}(i) \cdot \mathbf{x}
$$

where $y_{i}$ is the class-value of the training example $\mathbf{x}(i)$. The problem of finding the support vectors and parameters $b$ and $\left\{\alpha_{i}\right\}$ can be transformed into a linearly constrained quadratic programming $(\mathrm{QP})$ problem.

For the linearly separable case, we assume that all data is at least distance 1 from the hyperplane $w^{T} x_{i}+b=0$. Then, given a training set of instance-label pairs $\left(x_{i}, y_{i}\right)$, where $x_{i} \in R^{n}$ and $y_{i} \in\{1,-1\}$, the data points will be correctly classified by

$$
y_{i}\left(w^{T} x_{i}+b\right) \geq 1
$$

The SVM finds an optimal separating hyperplane with the maximum margin by solving the following quadratic optimization problem:

$$
\begin{aligned}
& \operatorname{Min} \Phi(w)=\frac{1}{2} w^{T} w \\
& \text { Subject to } y_{i}\left(w^{T} x_{i}+b\right) \geq 1
\end{aligned}
$$

By adopting nonnegative slack variables, we can transform (6) into (7) as follows:

$$
\begin{aligned}
& \operatorname{Min} \Phi(w)=\frac{1}{2} w^{T} w+C \sum_{i=1}^{m} \xi_{i} \\
& \text { Subject to } y_{i}\left(w^{T} x_{i}+b\right)+\xi_{i} \geq 1 \\
& \xi_{i} \geq 0 .
\end{aligned}
$$

By solving (7), we can find the hyperplane that provides the minimum number of training errors. For the nonlinear separable case, SVM models are able to undertake the classification by constructing a linear model that implements the nonlinear class boundaries by transforming the inputs into the high-dimensional feature space. In this case, (4) can be modified into a high-dimensional version as follows:

$$
y=b+\sum_{i=1}^{n} \alpha_{i} y_{i} K(\mathbf{x}(i) \cdot \mathbf{x}) .
$$

The function $K(\mathbf{x}(i) \cdot \mathbf{x})$ is the kernel function which transforms the input vector into a high-dimensional feature space. Usually, there are 3 types of kernel functions: the linear kernel, $K(x, y)=x^{T} y$; the polynomial kernel, $K(x, y)=\left(x^{T} y+1\right)^{d}$, where $d$ is the degree of the polynomial kernel; the Gaussian radial basis function $(\mathrm{RBF}), K(x, y)=\exp \left(-1 / \sigma^{2}(x-y)^{2}\right)$, where $\sigma^{2}$ is the bandwidth of the kernel.

2.3. Back-Propagation Neural Networks. Also, in the problem of financial forecasting, the technique of back-propagation neural networks (BPNN) is always used as a benchmark model [35-38]. The procedure to set up a BPNN includes the following: (1) select input and output variables; (2) determine layers and number of neurons in hidden layers; (3) learn from real data; (4) test; (5) recall. Let $q$ be the number of hidden nodes and $p$ the dimension of the input vector (the lagged observations). The relationship between the output $\left(y_{t}\right)$ and the inputs $\left(x_{T}\right)$ has the following mathematical representation:

$$
y_{t}=\alpha_{0}+\sum_{j=1}^{q} \alpha_{j} g\left(\beta_{0 j}+\sum_{i=1}^{p} \beta_{i j} x_{T i}\right)+\varepsilon_{t},
$$

where $\alpha_{j}(j=0,1,2, \ldots, q)$ is the connection weight of the $j$ th hidden node and $\beta_{i j}(i=0,1,2, \ldots, p)$ is the connection weight between the $i$ th input node and the $j$ th hidden node. The logistic function is often used as the hidden layer transfer function as follows:

$$
g(x)=\frac{1}{1+\exp (-x)} .
$$


TABLE 4: Normalized value of variables in period $T-1$.

\begin{tabular}{|c|c|c|c|c|c|c|c|c|c|c|c|}
\hline$x_{1}$ & $x_{2}$ & $x_{3}$ & $x_{4}$ & $x_{5}$ & $x_{6}$ & $x_{7}$ & $x_{8}$ & $x_{9}$ & $x_{10}$ & $x_{11}$ & $x_{12}$ \\
\hline 0.5252 & .6029 & 0.2373 & 0.4953 & 0.9818 & 0.8224 & 0.9074 & -0.5109 & 0.3745 & -0.9789 & -0.9592 & -0.2955 \\
\hline 0.3846 & 0.5393 & 0.2000 & 0.2906 & -0.9729 & -0.6107 & -0.9661 & -0.5505 & 0.1540 & -0.9826 & -0.9803 & -0.3119 \\
\hline 0.3939 & 0.5456 & 0.1573 & 0.4192 & -0.9923 & -0.8256 & -0.9833 & 0.5452 & -0.7498 & -0.9126 & -0.8648 & -0.2649 \\
\hline 0.3949 & 0.4314 & -0.1040 & 0.2464 & -0.9912 & -0.9068 & -0.9644 & 0.7426 & 0.1921 & -0.9742 & -0.9743 & -0.5082 \\
\hline 0.6351 & 0.6208 & 0.5253 & 0.4228 & -0.9759 & -0.4508 & -0.9718 & -0.3279 & 0.6683 & -0.9892 & -0.9881 & 0.1710 \\
\hline 0.5297 & 0.5918 & 0.3120 & 0.3372 & -0.9659 & -0.6025 & -0.9437 & -0.4944 & 0.1876 & -0.9894 & -0.9798 & -0.2916 \\
\hline 0.8457 & 0.5743 & 0.2107 & 0.3219 & -0.9747 & -0.6960 & -0.9564 & -0.4172 & -0.1122 & -0.9800 & -0.9730 & 0.0583 \\
\hline 0.3838 & 0.5146 & 0.2267 & 0.2580 & -0.9823 & -0.9529 & -0.9759 & -0.6413 & -0.2049 & -0.9745 & -0.9661 & -0.5825 \\
\hline 0.5171 & 0.6450 & 0.3067 & 0.4206 & -0.9706 & -0.7358 & -0.9692 & -0.5314 & -0.6949 & -0.9005 & -0.8373 & -0.0307 \\
\hline 0.5677 & 0.6253 & 0.5200 & 0.3072 & -0.9376 & -0.6906 & -0.9295 & -0.5577 & 0.0421 & -0.9720 & -0.9551 & -0.1686 \\
\hline 0.9948 & 1.0000 & 0.4347 & 0.5575 & -0.9476 & -0.8587 & -0.9227 & -0.5729 & -0.0191 & -0.9670 & -0.9436 & 0.3463 \\
\hline-0.0818 & 0.4718 & -0.2213 & 0.0015 & -0.9865 & -0.9213 & -0.9779 & -0.6634 & 0.6165 & -0.9912 & -0.9872 & -0.3183 \\
\hline 0.4188 & 0.5536 & 0.1733 & 0.2848 & -0.9706 & -0.4318 & -0.9601 & -0.8760 & 0.1072 & -0.9764 & -0.9675 & -0.3193 \\
\hline 0.4711 & 0.5776 & 0.2320 & 0.2985 & -0.9382 & -0.7292 & -0.8632 & -0.5895 & -0.4626 & -0.9443 & -0.8942 & -0.0677 \\
\hline 0.4593 & 0.5680 & 0.2693 & 0.3418 & -0.9818 & -0.7090 & -0.9897 & -0.7367 & 0.0073 & -0.9670 & -0.9666 & -0.1915 \\
\hline-1.0000 & -0.8001 & -0.2800 & 0.0370 & -1.0000 & -1.0000 & -0.9868 & -0.8257 & 0.3038 & -1.0000 & -0.9959 & -0.5343 \\
\hline 0.6446 & 0.6834 & 0.4027 & 0.3722 & -0.9653 & -0.8695 & -0.9620 & -0.5497 & 0.2582 & -0.9806 & -0.9 & -0.0290 \\
\hline 0.6328 & 0.7194 & 0.2853 & 0.3474 & -0.9335 & -0.5333 & -0.9137 & -0 & 0.2913 & -0 & -0 & -0 \\
\hline 0.5460 & 0.5942 & 0.4773 & 0.4068 & -0.9717 & -0.8562 & -0.9817 & -0.5383 & -0.1348 & -0.9672 & -0.5 & 0.1675 \\
\hline 0.0768 & 0.4780 & -0.1680 & -0.2569 & -0.9 & -0.9 & -0.9 & & & -0.9 & & -0.4178 \\
\hline 0.4535 & 0 & 0.24 & 3 & -0.9 & -0.8 & -0.9 & & & -0 & -0. & -0.2526 \\
\hline 0.4170 & 0.5674 & 0.2213 & 0.2874 & -0.9 & -0.7760 & -0.9660 & -0.6050 & 0.0991 & -0.9824 & -0.9762 & -0.2414 \\
\hline 0.6446 & 0.6462 & 0.4027 & 0.3722 & -0.9653 & -0.8695 & -0.9620 & -0.5505 & 0.2617 & -0.9806 & -0.9702 & 0.3556 \\
\hline 0.8959 & 0.4603 & -0.0933 & -1.0000 & -0.9882 & -0.9520 & -0.9913 & 1.0000 & 0.7052 & -0.9811 & -0.9739 & -0.3499 \\
\hline 0.6901 & 0.8262 & 0.4507 & 0.3951 & -0.9141 & -0.6628 & -0.9669 & -0.5 & -0.1720 & -0.5 & -0 & 0.3804 \\
\hline 0.4598 & 0.5780 & 0.3013 & 0.3860 & -0.9871 & -0.9646 & -0.9801 & -0.5414 & 0.1562 & -0.9049 & -0.7924 & -0.1728 \\
\hline 0.4415 & & 1.0000 & 0.3658 & -0.97 & -0.9415 & & & & & & 825 \\
\hline 0.2118 & & & & & -0.9596 & & & & & & \\
\hline 1.0000 & 0.8375 & 0.4613 & 0.3615 & -0.8599 & 1.0000 & -0.9109 & -0.5882 & 0.3462 & -0.9835 & -0.9762 & 0.2486 \\
\hline 0.4606 & 0.5746 & 0.2800 & 0.3548 & 1.0000 & -0.9061 & -0.9672 & -0.5989 & 0.0231 & -0.9804 & -0.9656 & -0.2583 \\
\hline 0.4821 & 0.6021 & 0.3760 & 0.3015 & -0.9812 & -0.8423 & -0.9643 & -0.6511 & -0.2789 & -0.9575 & -0.9212 & -0.3718 \\
\hline-0.3599 & 0.3544 & -0.1253 & 1.0000 & -0.9894 & -0.9614 & -0.9942 & -0.5679 & 0.2111 & -0.9767 & -0.9762 & -0.1396 \\
\hline 0.4404 & 0.5663 & 0.2533 & 0.3736 & -0.9776 & -0.8436 & -0.9607 & -0.4990 & 0.0062 & -0.9848 & -0.9771 & -0.3169 \\
\hline 0.5003 & 0.6259 & 0.2587 & 0.4123 & -0.9717 & -0.1673 & -0.9704 & -0.3763 & -0.6148 & -0.9165 & -0.8749 & 0.0821 \\
\hline 0.1803 & 0.4450 & 0.7067 & 0.1812 & -0.9370 & -0.6872 & -0.9066 & & 0.3669 & -0.9833 & -0.9702 & -0.2199 \\
\hline 0.2026 & 0.4579 & -0.1360 & 0.4088 & -0.9829 & -0.9450 & -0.9624 & -0.8518 & -0.2860 & -0.9685 & -0.9464 & -0.2119 \\
\hline 0.4372 & 0.5513 & 0.3280 & 0.2743 & -0.9329 & -0.5977 & -0.8275 & -0.6905 & -0.0322 & -0.9668 & -0.9381 & -0.3296 \\
\hline 0.3829 & 0.5286 & 0.1520 & 0.2560 & -0.9706 & -0.8670 & -0.9787 & -0.5989 & 0.3456 & -0.9817 & -0.9789 & -0.4399 \\
\hline 0.5111 & 0.6587 & 0.6053 & 0.4279 & -0.9741 & -0.7263 & -0.9756 & -0.6219 & -0.4752 & -0.8809 & -0.7892 & 0.2208 \\
\hline 0.3263 & -1.0000 & 0.1520 & 0.1989 & -0.9835 & -0.9245 & -0.9555 & -0.5935 & -0.8093 & -0.8604 & -0.6312 & -0.3272 \\
\hline 0.5063 & 0.6185 & 0.3280 & 0.3736 & -0.9659 & -0.8120 & -0.9506 & -0.5913 & -1.0000 & -0.9260 & -0.8777 & 0.1621 \\
\hline 0.6375 & 0.6612 & 0.5307 & 0.4582 & -0.9723 & -0.5917 & -0.9460 & -0.5641 & -0.9390 & -0.8714 & -0.8955 & -1.0000 \\
\hline 0.3818 & 0.5225 & 0.2480 & 0.2570 & -0.9723 & -0.7722 & 1.0000 & -0.5171 & 0.5616 & 1.0000 & 1.0000 & -0.4788 \\
\hline-0.8794 & 0.4366 & -0.1680 & -0.0156 & -0.9788 & -0.7020 & -0.9492 & -0.6940 & 0.0886 & -0.9846 & -0.9693 & -0.3795 \\
\hline 0.4724 & 0.5999 & 0.4667 & 0.2515 & -0.9641 & -0.8979 & -0.9658 & -1.0000 & -0.4520 & -0.9476 & -0.9102 & -0.3981 \\
\hline 0.6454 & 0.6612 & 0.6160 & 0.4437 & -0.9723 & -0.8761 & -0.9664 & -0.4732 & -0.0064 & -0.9643 & -0.9404 & -0.1961 \\
\hline 0.3758 & 0.5268 & 0.1467 & 0.2851 & -0.9935 & -0.8543 & -0.9802 & -0.5580 & 0.0599 & -0.9760 & -0.9505 & -0.3126 \\
\hline 0.0483 & 0.5086 & -0.2800 & 0.1874 & -0.9741 & -0.7466 & -0.9623 & -0.8562 & 0.4431 & -0.9850 & -0.9757 & -0.2344 \\
\hline 0.4042 & 0.5431 & 0.2000 & 0.2912 & -0.9741 & -0.7706 & -0.9207 & -0.5894 & -0.4615 & -0.9685 & -0.9446 & -0.2653 \\
\hline 0.5237 & 0.6053 & 0.3920 & 0.3593 & -0.9706 & -0.8673 & -0.9834 & -0.6655 & -0.0140 & -0.9683 & -0.9730 & -0.2009 \\
\hline
\end{tabular}


TABle 4: Continued.

\begin{tabular}{lccccccccccc}
\hline$x_{1}$ & $x_{2}$ & $x_{3}$ & $x_{4}$ & $x_{5}$ & $x_{6}$ & $x_{7}$ & $x_{8}$ & $x_{9}$ & $x_{10}$ & $x_{11}$ & $x_{12}$ \\
\hline 0.4299 & 0.5779 & 0.2213 & 0.3224 & -0.9700 & -0.8300 & -0.9380 & -0.5264 & 0.2163 & -0.8648 & -0.7714 & -0.2456 \\
0.6310 & 0.5331 & 0.2160 & 0.1410 & -0.9906 & -0.8891 & -0.9770 & -0.5247 & -0.8744 & -0.9866 & -1.0000 & -0.3269 \\
-0.1204 & 0.5166 & 0.0613 & 0.1796 & -0.9747 & -0.8600 & -0.9492 & -0.5776 & 0.7844 & -0.9938 & -0.9890 & -0.3867 \\
0.4913 & 0.5978 & 0.2800 & 0.3384 & -0.9617 & -0.7033 & -0.9327 & -0.5668 & -0.3724 & -0.9769 & -0.9675 & -0.1122 \\
0.5630 & 0.6660 & 0.6427 & 0.6103 & -0.9794 & -0.6505 & -0.9864 & -0.4540 & -0.6894 & -0.8778 & -0.7856 & -0.2509 \\
0.0069 & 0.2051 & -1.0000 & 0.2103 & -0.9971 & -0.9542 & -1.0000 & -0.0220 & 0.9151 & -0.9780 & -0.9592 & -0.7189 \\
0.4389 & 0.5745 & 0.4293 & 0.2974 & -0.9612 & -0.7693 & -0.9681 & -0.6451 & -0.1182 & -0.9676 & -0.9588 & -0.2383 \\
0.3799 & 0.5339 & 0.1520 & 0.2646 & -0.9706 & -0.7791 & -0.9541 & -0.6428 & -0.1148 & -0.9767 & -0.9656 & -0.2599 \\
0.3904 & 0.5408 & 0.1573 & 0.2708 & -0.9818 & -0.7301 & -0.9460 & -0.5311 & 0.0297 & -0.9855 & -0.9716 & -0.2828 \\
0.6699 & 0.6805 & 0.3333 & 0.4646 & -0.9653 & -0.9302 & -0.9707 & -0.5662 & -0.1437 & -0.9604 & -0.9120 & 1.0000 \\
\hline
\end{tabular}

The BPNN model performs a nonlinear functional mapping from inputs $\left(x_{T}\right)$ to $y_{t}$ as follows:

$$
y_{t}=f\left(x_{T}, \theta\right)+\varepsilon_{t}
$$

where $\theta$ is a vector of all parameters and $f$ is a function determined by the network structure and connection weights. Thus, the neural network is equivalent to a nonlinear autoregressive model.

However, individual methods for corporate financial distress forecasting have a disadvantage for error recognition. Therefore, in the following section, a novel ensemble learning approach based on logistic regression and SVM/BPNN is proposed for error recognition and the improvement of forecasting performance.

\section{A Novel Ensemble Learning Approach}

Many hybrid methods have been used for economic forecasting [39-41], and risk analysis has been addressed in supply chain management [42-48]. When the methods of LR, SVM, and BPNN are used for corporate financial distress forecasting, financial ratio indices of companies are input variables and corresponding corporate financial state $(0 / 1)$ of companies is the output. The principle of the new ensemble learning approach based on LR and SVM/BPNN is that the forecasting results of corporate financial distress by LR are set as another input variable within the SVM/BPNN framework, corresponding to the output of corporate financial state. In this way, both Type I error (reject-true error) and Type II error (accept-false error) in LR analysis can be recognized by SVM/BPNN.

In summary, the overall process of the ensemble learning approach (LR-SVM/BPNN) can be described in Figure 1 in the following three main steps.

(1) Stepwise regression analysis is used to remove independent variables which are insignificantly linear with the dependent variable. In this way, remained independent variables are significant to the dependent variable and multicollinearity is removed.

(2) Logistic regression analysis is implemented based on train sample set. Then, critical value of financial distress probability is set as 0.5 . If the probability is

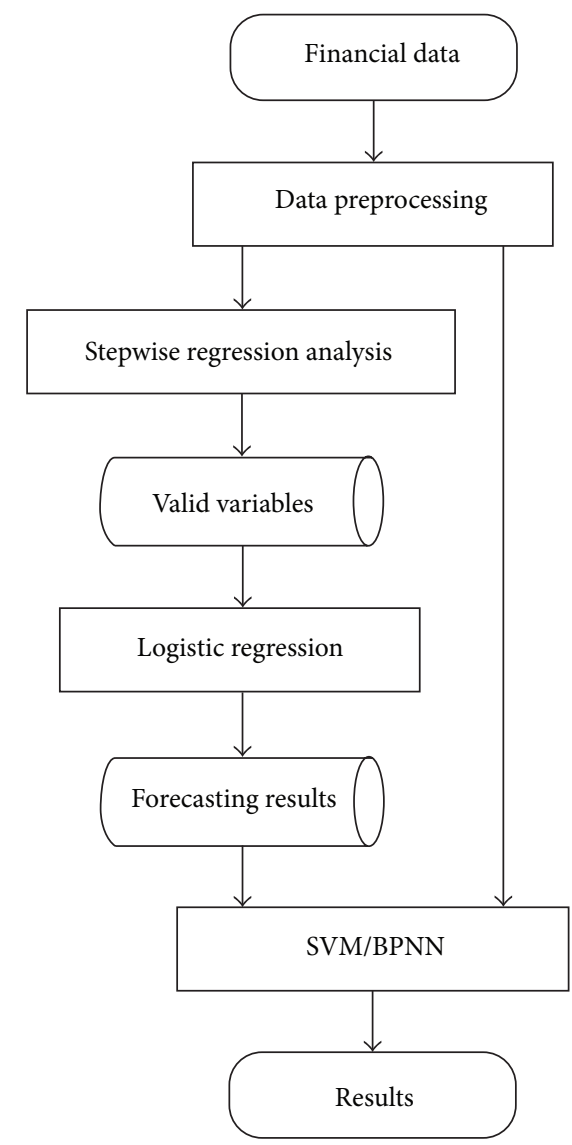

FIGURE 1: Framework of the proposed ensemble learning approach.

more than 0.5 , the value is 1 and the company is predicted to have financial distress; or else, the value is 0 and the company is predicted to have no financial distress.

(3) The forecasting results by LR are introduced into the SVM/BPNN technique as a new variable. Then, there will be a new forecasting result by the LR-SVM/BPNN approach.

On the whole, Logistic regression is a linear model, while $\mathrm{SVM} / \mathrm{BPNN}$ is nonlinear model. When the forecasting results 
TABLE 5: Comparison of forecasting performance with variables in period $T$.

\begin{tabular}{lccccc}
\hline & LR & BPNN & SVM & LR-BPNN & LR-SVM \\
\hline Type I error rate & $46.7 \%$ & $20 \%$ & $13.3 \%$ & $13.3 \%$ & $6.7 \%$ \\
Type II error rate & $40 \%$ & $20 \%$ & $40 \%$ & $20 \%$ & $40 \%$ \\
Total accuracy & $55 \%$ & $80 \%$ & $80 \%$ & $85 \%$ & $85 \%$ \\
\hline
\end{tabular}

TABLE 6: Comparison of forecasting performance with variables in period $T-2$.

\begin{tabular}{lccccc}
\hline & LR & BPNN & SVM & LR-BPNN & LR-SVM \\
\hline Type I error rate & $46.7 \%$ & $33.3 \%$ & $26.7 \%$ & $26.7 \%$ & $13.3 \%$ \\
Type II error rate & $60 \%$ & $40 \%$ & $40 \%$ & $20 \%$ & $40 \%$ \\
Total accuracy & $50 \%$ & $65 \%$ & $70 \%$ & $75 \%$ & $80 \%$ \\
\hline
\end{tabular}

TABLE 7: Comparison of forecasting performance with variables in period $T-3$.

\begin{tabular}{lccccc}
\hline & LR & BPNN & SVM & LR-BPNN & LR-SVM \\
\hline Type I error rate & $53.3 \%$ & $33.3 \%$ & $26.7 \%$ & $20 \%$ & $26.7 \%$ \\
Type II error rate & $60 \%$ & $60 \%$ & $40 \%$ & $40 \%$ & $20 \%$ \\
Total accuracy & $45 \%$ & $60 \%$ & $70 \%$ & $75 \%$ & $75 \%$ \\
\hline
\end{tabular}

by LR are introduced into the SVM/BPNN technique as an input variable, SVM/BPNN can recognize the forecasting errors in fitness by LR. Therefore, the ensemble learning approach LR-SVM/BPNN is promising to achieve better forecasting performance.

\section{Empirical Analysis}

4.1. Data Description and Experiment Design. Fashion and textile sector is a traditional main industry in China. In recent years, China's export in fashion and textile sector is more than $25 \%$ in the global trade. However, many fashion and textile companies have been shocked immensely since the financial crisis in 2008. When a fashion and textile company suffers from financial distress, other companies in its supply chains will be subjected to financial risk. Therefore, financial distress forecasting is important in fashion and textiles supply chains.

In this study, the data for our experiment were collected from the Shanghai Stock Exchange and Shenzhen Stock Exchange databases in China. In 2012, there are 88 fashion and textiles related listed companies such as Shenzhen Victor Onward Textile Industrial Co., Ltd. (000018); Xinlong Holding (Group) Company Ltd. (000955); Hubei Maiya Co., Ltd. (000971); Shandong Demian Incorporated Company (002072); Lanzhou Sanmao Industrial Co., Ltd. (000779); Ningxia Zhongyin Cashmere Co. Ltd. (000982); Sichuan Langsha Holding Ltd. (600137); Hunan Huasheng Co., Ltd. (600156); Xinjiang Tianshan Wool Tex Stock Co., Ltd. (000813); Nanjing Textiles Import \& Export Corp., Ltd. (600250); Henan Xinye Textile Co., Ltd. (002087), where numbers in brackets are stock codes of corresponding listed companies. We selected 15 companies once special-treated (ST) and 45 non-ST companies as samples, where ST are regarded as financial distress in this study. Also, we selected 12 variables and categorized them as four major types: earning ability, operating ability, debt-repaying ability, and growing ability. The details of these indicators belong to each type and are listed in Table 1.

The data set consisting of 60 samples are listed in Table 2, where variable $y$ represents financial distress state $(0$ means no financial distress and 1 means financial distress) in period $T$ and $x_{i}(i=1,2, \ldots, 12)$ represents the value of the $i$ th variable in period $T-1$. In this study, $x_{i}$ is an independent variable and $y$ is a dependent variable.

The training set (first 40 samples in Table 2) and the testing set (last 20 samples in Table 2) used in empirical analysis are described in Table 3. Here, the training set is used to acquire the parameters of forecasting models, while the testing set is used to measure the forecasting performance of forecasting models.

4.2. Experiment Results and Analysis. In the empirical analysis of corporate financial distress forecasting, financial state of listed companies in period $T$ is dependent variable/output and variables in period $T-1$ are input. Normalization of variables in period $T-1$ is implemented and the normalized value is listed in Table 4 .

As a result, the methods are used for corporate financial distress forecasting in the next period when the value of variables in current period are available. By using the training set and the testing set in Table 3, we obtain the forecasting performance comparison of methods with values of variables in period $T-1$ and financial state in period $T$ as in Table 5 .

Moreover, independent variable values in periods $T-2$ and $T-3$ are used for corporate financial distress (independent variable in period $T$ ) forecasting, and comparison of forecasting performance with period $T-2$ and period $T-3$ data is shown in Tables 6 and 7.

From the results in Table 5, the accuracy achieved by BPNN and SVM is $80 \%$, and that by LR-BPNN and LR-SVM is $85 \%$, which is much higher than the accuracy $50 \%$ by LR. In addition, by comparing the results in Tables 5-7, we can find that the total accuracy decreases in the length from current period to period $T$; that is, total accuracy in period $T-1$ is better than that in period $T-2$, which is better than that in period $T-3$. Therefore, we can conclude that artificial intelligence techniques, that is, SVM and BPNN, can achieve better forecasting performance than LR. In addition, as SVM/BPNN can recognize the forecasting errors in fitness by LR, ensemble learning approaches LR-BPNN and LR-SVM can achieve better forecasting performance than individual methods when the forecasting results by LR are introduced into the SVM/BPNN technique as an input variable.

\section{Conclusions and Future Work}

In this study, logistic regression (LR) model is integrated with artificial intelligence tools, that is, support vector machine (SVM) and back-propagation neural networks (BPNN), for corporate financial distress forecasting in fashion and textiles supply chains. Empirical analysis of Chinese listed companies 
in fashion and textile sector is implemented for the comparison of the methods, and some related issues are discussed.

The contribution of this study is that a novel ensemble learning approach is developed for corporate financial distress forecasting in fashion and textiles supply chains. In the framework of the proposed ensemble learning approach, the forecasting results by LR are introduced into the SVM and BPNN techniques which can recognize the forecasting errors in fitness by LR. The results suggest that artificial intelligence tools are better than LR and the proposed novel ensemble learning approach can achieve better forecasting performance than that of individual models. By using the proposed approach, managers in fashion and textiles companies can predict the financial state of their suppliers, manufacturers, and retailers in advance and give a quick response for better supply chain performance.

It is expected that future research would benefit from the concentration on other methods for corporate financial distress forecasting, using data from a wider sample of fashion and textiles companies.

\section{Acknowledgments}

The authors sincerely thank the anonymous referees for their valuable suggestions and comments. This work was supported by the National Natural Science Foundation of China (Grant no. 70871107 and 71101028), China Postdoctoral Science Foundation (Grant no. 20060400103), the Program for Innovative Research Team in UIBE, the Program for Excellent Talents, UIBE and The Royal Academy of Engineering for research exchanges with China and India scheme.

\section{References}

[1] M. Christopher, R. Lowson, and H. Peck, "Creating agile supply chains in the fashion industry," International Journal of Retail \& Distribution Management, vol. 32, no. 8, pp. 367-376, 2004.

[2] M. Bruce, L. Daly, and N. Towers, "Lean or agile: a solution for supply chain management in the textiles and clothing industry?" International Journal of Operations and Production Management, vol. 24, no. 1-2, pp. 151-170, 2004.

[3] M. P. de Brito, V. Carbone, and C. M. Blanquart, "Towards a sustainable fashion retail supply chain in Europe: organisation and performance," International Journal of Production Economics, vol. 114, no. 2, pp. 534-553, 2008.

[4] P. Hilletofth and O. P. Hilmola, "Supply chain management in fashion and textile industry," International Journal of Services Sciences, vol. 1, no. 2, pp. 127-147, 2008.

[5] S. Thomassey, "Sales forecasts in clothing industry: the key success factor of the supply chain management," International Journal of Production Economics, vol. 128, no. 2, pp. 470-483, 2010.

[6] L. Barnes and G. Lea-Greenwood, "Fast fashioning the supply chain: shaping the research agenda," Journal of Fashion Marketing and Management, vol. 10, no. 3, pp. 259-271, 2006.

[7] G. Birtwistle, N. Siddiqui, and S. S. Fiorito, "Quick response: perceptions of UK fashion retailers," International Journal of Retail \& Distribution Management, vol. 31, no. 2, pp. 118-128, 2003.
[8] S. G. Hayes and N. Jones, "Fast fashion: a financial snapshot," Journal of Fashion Marketing and Management, vol. 10, no. 3, pp. 282-300, 2006.

[9] Z. L. Sun, T. M. Choi, K. F. Au, and Y. Yu, "Sales forecasting using extreme learning machine with applications in fashion retailing," Decision Support Systems, vol. 46, no. 1, pp. 411-419, 2008.

[10] T. M. Choi, Y. Yu, and K. F. Au, "A hybrid SARIMA wavelet transform method for sales forecasting," Decision Support Systems, vol. 51, no. 1, pp. 130-140, 2011.

[11] Y. Yu, T. M. Choi, and C. L. Hui, "An intelligent fast sales forecasting model for fashion products," Expert Systems with Applications, vol. 38, no. 6, pp. 7373-7379, 2011.

[12] K. F. Au, T. M. Choi, and Y. Yu, "Fashion retail forecasting by evolutionary neural networks," International Journal of Production Economics, vol. 114, no. 2, pp. 615-630, 2008.

[13] Y. Yu, C. L. Hui, and T. M. Choi, "An empirical study of intelligent expert systems on forecasting of fashion color trend," Expert Systems With Applications, vol. 39, pp. 4383-4389, 2012.

[14] T. M. Choi, C. L. Hui, S. F. F. Ng, and Y. Yu, "Color trend forecasting of fashionable products with very few historical data," IEEE Transactions on Systems, Man, and Cybernetics, Part C, vol. 42, pp. 1003-1010, 2012.

[15] Z. Hua, Y. Wang, X. Xu, B. Zhang, and L. Liang, "Predicting corporate financial distress based on integration of support vector machine and logistic regression," Expert Systems with Applications, vol. 33, no. 2, pp. 434-440, 2007.

[16] W. Beaver, "Financial ratios as predictors of failure, empirical research in accounting: selected studied," Journal of Accounting Research, vol. 4, pp. 71-111, 1968.

[17] E. L. Altman, "Financial ratios, discriminant analysis and the prediction of corporate bankruptcy," The Journal of Finance, vol. 23, no. 3, pp. 589-609, 1968.

[18] M. Blum, "Failing company discriminant analysis," Journal of Accounting Research, vol. 12, no. 1, pp. 1-25, 1974.

[19] E. I. Altman, R. G. Haldeman, and P. Narayanan, "ZETATM analysis A new model to identify bankruptcy risk of corporations," Journal of Banking and Finance, vol. 1, no. 1, pp. 29-54, 1977.

[20] P. A. Meyer and H. Pifer, "Prediction of bank failures," The Journal of Finance, vol. 25, pp. 853-868, 1970.

[21] E. K. Laitinen and T. Laitinen, "Bankruptcy prediction: application of the Taylor's expansion in logistic regression," International Review of Financial Analysis, vol. 9, no. 4, pp. 327-349, 2000.

[22] A. I. Dimitras, S. H. Zanakis, and C. Zopounidis, "A survey of business failures with an emphasis on prediction methods and industrial applications," European Journal of Operational Research, vol. 90, no. 3, pp. 487-513, 1996.

[23] M.-Y. Chen, "Predicting corporate financial distress based on integration of decision tree classification and logistic regression," Expert Systems with Applications, vol. 38, no. 9, pp. 1126111272,2011

[24] W.-S. Chen and Y. K. Du, "Using neural networks and data mining techniques for the financial distress prediction model," Expert Systems with Applications, vol. 36, no. 2, pp. 4075-4086, 2009.

[25] H. Li and J. Sun, "Ranking-order case-based reasoning for financial distress prediction," Knowledge-Based Systems, vol. 21, no. 8, pp. 868-878, 2008. 
[26] H. Li and J. Sun, "Gaussian case-based reasoning for business failure prediction with empirical data in China," Information Sciences, vol. 179, no. 1-2, pp. 89-108, 2009.

[27] K. Kim and H. Ahn, "A corporate credit rating model using multi-class support vector machines with an ordinal pairwise partitioning approach," Computers \& Operations Research, vol. 39, no. 8, pp. 1800-1811, 2012.

[28] J. Sun and H. Li, "Financial distress prediction using support vector machines: ensemble versus individual," Applied Soft Computing, vol. 12, pp. 2254-2265, 2012.

[29] C.-J. Lu, T.-S. Lee, and C.-C. Chiu, "Financial time series forecasting using independent component analysis and support vector regression," Decision Support Systems, vol. 47, no. 2, pp. 115-125, 2009.

[30] Z. Xiao, X. Yang, Y. Pang, and X. Dang, "The prediction for listed companies' financial distress by using multiple prediction methods with rough set and Dempster-Shafer evidence theory," Knowledge-Based Systems, vol. 26, pp. 196-206, 2012.

[31] J. Sun, M. Y. Jia, and H. Li, "AdaBoost ensemble for financial distress prediction: an empirical comparison with data from Chinese listed companies," Expert Systems with Applications, vol. 38, no. 8, pp. 9305-9312, 2011.

[32] H. Li and J. Sun, "Empirical research of hybridizing principal component analysis with multivariate discriminant analysis and logistic regression for business failure prediction," Expert Systems with Applications, vol. 38, no. 5, pp. 6244-6253, 2011.

[33] D. W. Hosmer and S. Lemeshow, Applied Logistic Regression, Wiley, New York, NY, USA, 2000.

[34] V. N. Vapnik, The Nature of Statistical Learning Theory, Springer, New York, NY, USA, 1995.

[35] P. G. Harrald and M. Kamstra, "Evolving artificial neural networks to combine financial forecasts," IEEE Transactions on Evolutionary Computation, vol. 1, no. 1, pp. 40-52, 1997.

[36] C. Hamzaçebi, D. Akay, and F. Kutay, "Comparison of direct and iterative artificial neural network forecast approaches in multi-periodic time series forecasting," Expert Systems with Applications, vol. 36, no. 2, pp. 3839-3844, 2009.

[37] J. V. Hansen and R. D. Nelson, "Neural networks and traditional time series methods: a synergistic combination in state economic forecasts," IEEE Transactions on Neural Networks, vol. 8, no. 4, pp. 863-873, 1997.

[38] W. Leigh, R. Purvis, and J. M. Ragusa, "Forecasting the NYSE composite index with technical analysis, pattern recognizer, neural network, and genetic algorithm: a case study in romantic decision support," Decision Support Systems, vol. 32, no. 4, pp. 361-377, 2002.

[39] B. Majhi, M. Rout, R. Majhi, G. Panda, and P. J. Fleming, "New robust forecasting models for exchange rates prediction," Expert Systems with Applications, vol. 39, no. 16, pp. 12658-12670, 2012.

[40] Z. L. Sun, K. F. Au, and T. M. Choi, "A neuro-fuzzy inference system through integration of fuzzy logic and extreme learning machines," IEEE Transactions on Systems, Man, and Cybernetics B, vol. 37, no. 5, pp. 1321-1331, 2007.

[41] B. Premanode and C. Toumazou, "Improving prediction of exchange rates using differential EMD," Expert Systems with Applications, vol. 40, no. 1, pp. 377-384, 2013.

[42] G. Xie, W. Yue, W. Liu, and S. Wang, "Risk based selection of cleaner products in a green supply chain," Pacific Journal of Optimization, vol. 8, no. 3, pp. 473-484, 2012.

[43] G. Xie, S. Wang, and K. K. Lai, "Quality improvement in competing supply chains," International Journal of Production Economics, vol. 134, no. 1, pp. 262-270, 2011.
[44] G. Xie, W. Yue, S. Wang, and K. K. Lai, "Quality investment and price decision in a risk-averse supply chain," European Journal of Operational Research, vol. 214, no. 2, pp. 403-410, 2011.

[45] G. Xie, S. Wang, and K. K. Lai, "Optimal $\beta_{k}$-stable interval in VPRS-based group decision-making: a further application," Expert Systems with Applications, vol. 38, no. 11, pp. 13757-13763, 2011.

[46] G. Xie, W. Yue, S. Wang, and K. K. Lai, "Dynamic risk management in petroleum project investment based on a variable precision rough set model," Technological Forecasting \& Social Change, vol. 77, no. 6, pp. 891-901, 2010.

[47] G. Xie, W. Yue, and S. Wang, "VPRS-based group decisionmaking for risk response in petroleum investment," International Journal of Knowledge and Systems Sciences, vol. 1, no. 3, pp. 45-54, 2010.

[48] G. Xie, J. Zhang, K. K. Lai, and L. Yu, "Variable precision rough set for group decision-making: an application," International Journal of Approximate Reasoning, vol. 49, no. 2, pp. 331-343, 2008. 


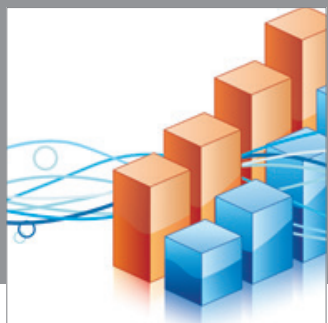

Advances in

Operations Research

mansans

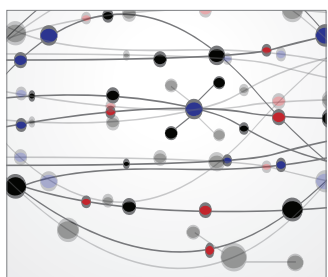

The Scientific World Journal
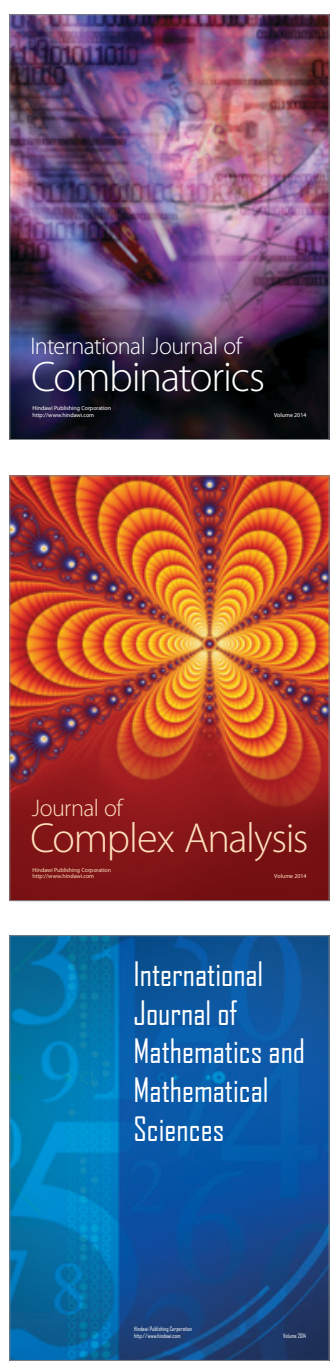
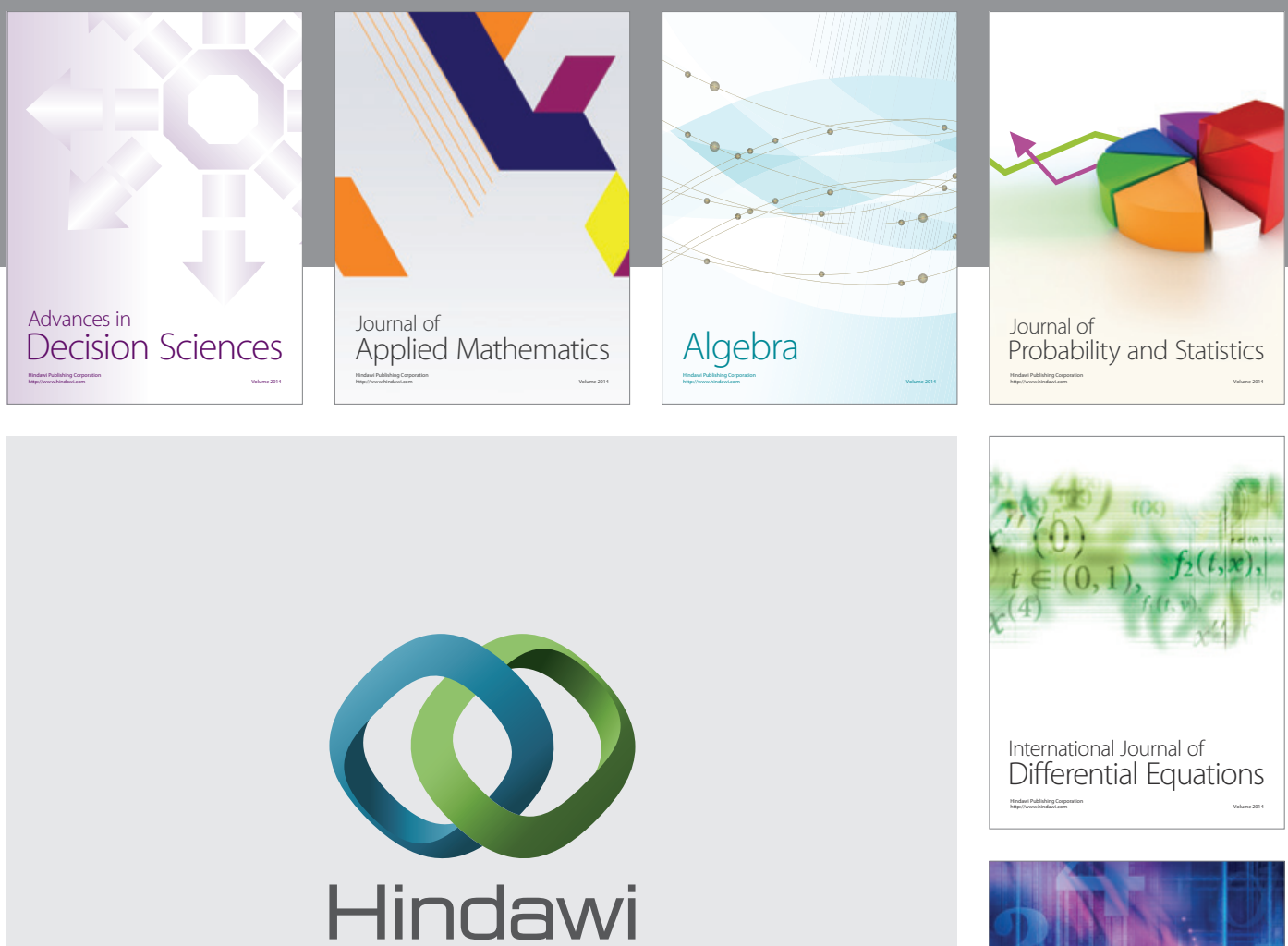

Submit your manuscripts at http://www.hindawi.com
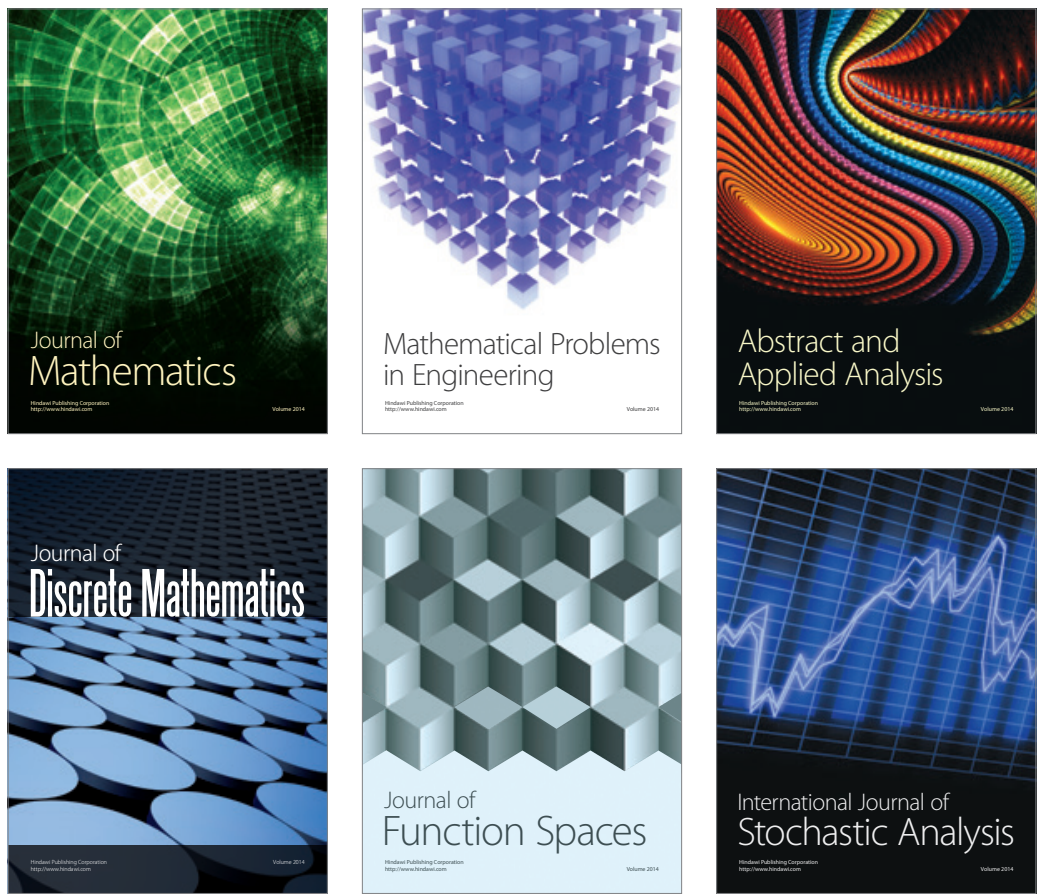

Journal of

Function Spaces

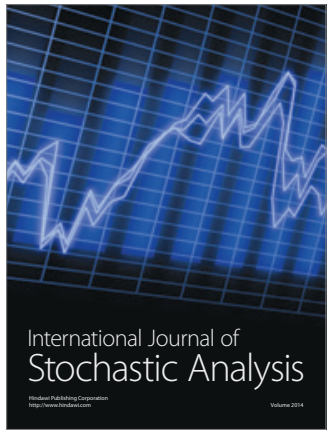

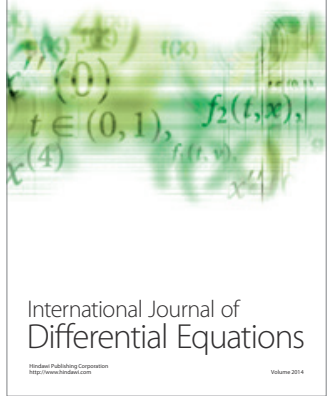
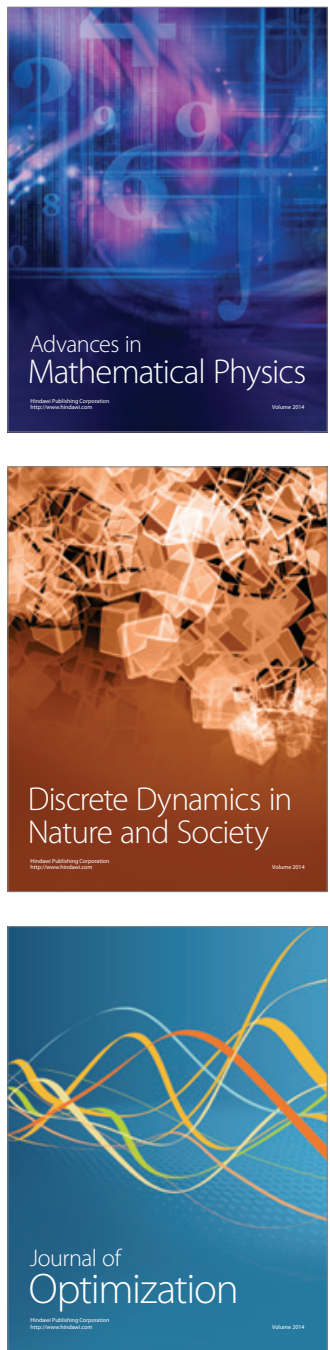